\title{
The extension theorem for bi-invariant weights over Frobenius rings and Frobenius bimodules
}

\author{
Oliver W. Gnilke, Marcus Greferath, Thomas Honold, Jay A. Wood, \\ and Jens Zumbrägel \\ In memory of our colleague and friend Aleksandr A. Nechaev, 1945-2014
}

\begin{abstract}
We give a sufficient condition for a bi-invariant weight on a Frobenius bimodule to satisfy the extension property. This condition applies to bi-invariant weights on a finite Frobenius ring as a special case. The complexvalued functions on a Frobenius bimodule are viewed as a module over the complex monoid algebra of the multiplicative monoid of the coefficient ring.
\end{abstract}

\section{Introduction}

When coding theory was first developed in the 1940s and 50s, linear codes were defined over finite fields using the Hamming weight. Coding theory has evolved since those early years, and linear codes are now often defined over alphabets that are finite modules over a finite ring using a more general weight on the module.

A fundamental question about linear codes, regardless of the level of abstraction, is: When should two linear codes be considered equivalent? There are two competing notions of equivalence. One notion is that two linear codes are equivalent if there exists a weight-preserving monomial transformation of the ambient space that takes one code to the other. The other notion is that two linear codes are equivalent if there exists a weight-preserving linear isomorphism between the two codes. If two linear codes are equivalent in the first sense, then they are equivalent in the second sense. Indeed, the restriction of the weight-preserving monomial transformation to the codes defines a weight-preserving linear isomorphism between them.

The converse, i.e., if two linear codes are equivalent in the second sense, then they are equivalent in the first sense, is called the extension problem, because the question is whether every weight-preserving linear isomorphism between two linear codes extends to a weight-preserving monomial transformation of the ambient space. When the extension problem for a given alphabet and a given weight has a positive solution, i.e., when every weight-preserving linear isomorphism extends to a weight-preserving monomial transformation, we often say that the extension theorem holds for that alphabet and weight.

The extension theorem is known to hold in a number of situations. The earliest version, over finite fields with the Hamming weight, is due to MacWilliams [10,11. The extension theorem holds when the alphabet is a finite Frobenius ring using the 
Hamming weight [3] or the homogeneous weight [7, and the extension theorem holds over Frobenius bimodules over any finite ring using either the Hamming or the homogeneous weight 6 .

There has been considerable work on determining tractable conditions on a general weight in order that the extension theorem hold for that weight. Some of the earliest work involved a nonsingularity condition on a matrix determined by both the alphabet and the weight that allowed one to reduce the problem to the known extension properties of a so-called symmetrized weight composition 14 .

This paper, drawing on ideas introduced in [2], 3] and [5, describes conditions on a weight with maximal symmetry (called a bi-invariant weight) over a Frobenius bimodule so that the extension problem reduces to the known extension properties of the homogeneous weight. The conditions found generalize those for weights defined over a finite principal ideal ring in [4, Theorem 4.4]. The reduction process is accomplished by viewing any weight on a Frobenius bimodule as an element of a complex vector space which is itself a module over the complex monoid algebra of the multiplicative monoid of the coefficient ring of the Frobenius bimodule. The additional algebraic structure of modules over monoid algebras allows us to compare the extension properties of weights that are scalar multiples over the monoid algebra. The key observation, dating from [2], is that if $w^{\prime}$ is a scalar multiple of $w$, both having maximal symmetry, and if the extension theorem holds for $w^{\prime}$, then the extension theorem holds for $w$. We apply this idea with $w^{\prime}$ equaling the homogeneous weight, and the conditions we determine are those that allow us to find a scalar $\gamma$ such that $w \gamma=w^{\prime}$.

Here is a short guide to the rest of the paper. Monoid algebras and certain modules are introduced in Section 2. Detailed computations, especially solving $w \gamma=w^{\prime}$ for $\gamma$, are carried out in Section 3. The proofs of the main results are in Section 4. The main theorem is illustrated by an example in Section 5. The paper concludes in Section [ with an extension of the main theorem to alphabets that can be viewed as pseudo-injective submodules of a Frobenius bimodule.

We thank the referee for helpful comments, especially for suggesting what became Lemma 3.7 .

\section{Preliminaries}

Let $R$ be a finite ring with 1 . Denote the group of units of $R$ by $\mathcal{U}$. Let $\widehat{R}$ be the group of complex characters of the additive group of $R$; i.e., $\widehat{R}=\left\{\pi: R \rightarrow \mathbb{C}^{\times}\right.$: $\left.\pi\left(r_{1}+r_{2}\right)=\pi\left(r_{1}\right) \pi\left(r_{2}\right), r_{1}, r_{2} \in R\right\}$. Then $\widehat{R}$ is an abelian group under point-wise multiplication of functions, and $\widehat{R}$ is a bimodule over $R$ under the following scalar multiplications: $\left({ }^{s} \pi\right)(r)=\pi(r s)$ and $\left(\pi^{s}\right)(r)=\pi(s r)$, for all $r, s \in R$.

Let $A$ be a Frobenius bimodule over $R$; i.e., $A$ is a bimodule over $R$ such that ${ }_{R} A \cong{ }_{R} \widehat{R}$ and $A_{R} \cong \widehat{R}_{R}$. In particular, $|A|=|R|$. The Frobenius bimodule $A$ admits a character $\chi$ that generates $\widehat{A}$ both as a left $R$-module and as a right $R$ module. This character $\chi$ also has the equivalent properties that ker $\chi$ contains no nonzero left nor right $R$-submodules. We will refer to $\chi$ as a generating character for $A$. Details concerning these results may be found in [15, Section 5.2].

LEMMA 2.1. If $S$ is a nonzero left or right submodule of $A$, then $\sum_{s \in S} \chi(s)=0$.

Proof. Because ker $\chi$ contains no nonzero left or right submodules, there is some $s_{0} \in S$ with $\chi\left(s_{0}\right) \neq 1$. Let $t=s+s_{0}$ be a change of variables in the given 
sum:

$$
\sum_{t \in S} \chi(t)=\sum_{s \in S} \chi\left(s+s_{0}\right)=\sum_{s \in S} \chi(s) \chi\left(s_{0}\right)=\chi\left(s_{0}\right) \sum_{s \in S} \chi(s) .
$$

Thus, $\left(1-\chi\left(s_{0}\right)\right) \sum_{s \in S} \chi(s)=0$, so that $\sum_{s \in S} \chi(s)=0$.

Let $\mathcal{R}$ denote the complex monoid algebra of the multiplicative monoid of $R$. Explicitly, $\mathcal{R}$ is the vector space of all functions $\mathcal{R}=\{\alpha: R \rightarrow \mathbb{C}\}$ from $R$ to the complex numbers $\mathbb{C}$, equipped with the multiplicative convolution product $*$ :

$$
(\alpha * \beta)(r)=\sum_{s t=r} \alpha(s) \beta(t),
$$

where the sum is over all pairs $(s, t) \in R \times R$ satisfying $s t=r$.

Let $\mathcal{A}=\{w: A \rightarrow \mathbb{C}\}$ be the vector space of all functions from $A$ to $\mathbb{C}$. For $w \in \mathcal{A}$ and $\alpha \in \mathcal{R}$, define the (right) multiplicative correlation $w \circledast \alpha \in \mathcal{A}$ by $(w \circledast \alpha)(a)=\sum_{r \in R} w(r a) \alpha(r)$, for $a \in A$. In a similar fashion, one can define a left multiplicative correlation; we will not need the left version in this paper.

Define a Fourier transform ${ }^{\wedge}: \mathcal{R} \rightarrow \mathcal{A}$ by $\widehat{\alpha}=\chi \circledast \alpha$; that is, for $a \in A$,

$$
\widehat{\alpha}(a)=\sum_{r \in R} \chi(r a) \alpha(r) .
$$

Lemma 2.2. The spaces $\mathcal{R}$ and $\mathcal{A}$ satisfy the following, with $w \in \mathcal{A}, \alpha, \beta \in \mathcal{R}$ :

(1) $\mathcal{R}$ is a finite-dimensional algebra over $\mathbb{C}$; $\operatorname{dim} \mathcal{R}=|R|$.

(2) $\mathcal{A}$ is a right $\mathcal{R}$-module under $\circledast: w \circledast(\alpha * \beta)=(w \circledast \alpha) \circledast \beta$.

$(3) \widehat{\widehat{A}}: \mathcal{R} \rightarrow \mathcal{A}$ is an isomorphism of right $\mathcal{R}$-modules; in particular, $\widehat{\alpha * \beta}=$ $\widehat{\alpha} \circledast \beta$. The inverse sends $w \in \mathcal{A}$ to $\widetilde{w}(r)=(1 /|A|) \sum_{a \in A} w(a) \chi(-r a)$.

Proof. These are routine verifications. We will show one of them.

$$
\begin{aligned}
((w \circledast \alpha) \circledast \beta)(a) & =\sum_{t \in R}(w \circledast \alpha)(t a) \beta(t) \\
& =\sum_{t \in R}\left(\sum_{s \in R} w(s t a) \alpha(s)\right) \beta(t) \\
& =\sum_{r \in R} \sum_{s t=r} w(r a) \alpha(s) \beta(t)=(w \circledast(\alpha * \beta))(a)
\end{aligned}
$$

For the inverse transform claim, see Lemma 3.1

We define subspaces of $\mathcal{R}$ and $\mathcal{A}$ : let $\mathcal{R}_{0}=\left\{\alpha \in \mathcal{R}: \sum_{r \in R} \alpha(r)=0\right\}$ and $\mathcal{A}_{0}=\{w \in \mathcal{A}: w(0)=0\}$.

Lemma 2.3. (1) For $\alpha, \beta \in \mathcal{R}$,

$$
\sum_{r \in R}(\alpha * \beta)(r)=\left(\sum_{s \in R} \alpha(s)\right)\left(\sum_{t \in R} \beta(t)\right) .
$$

(2) For $w \in \mathcal{A}$ and $\alpha \in \mathcal{R},(w \circledast \alpha)(0)=w(0) \sum_{r \in R} \alpha(r)$.

(3) For $\alpha \in \mathcal{R}, \widehat{\alpha}(0)=\sum_{r \in R} \alpha(r)$.

(4) $\mathcal{R}_{0}$ is a two-sided ideal in $\mathcal{R}$.

(5) $\mathcal{A}_{0}$ is a right $\mathcal{R}$-submodule of $\mathcal{A}$.

(6) $\widehat{\wedge}: \mathcal{R}_{0} \rightarrow \mathcal{A}_{0}$ is an isomorphism of right $\mathcal{R}$-modules.

Proof. Again, these are routine verifications. 
An element $\alpha \in \mathcal{R}$ is bi-invariant if $\alpha(u r v)=\alpha(r)$ for all $r \in R$ and $u, v \in \mathcal{U}$. Similarly, an element $w \in \mathcal{A}$ is bi-invariant if $w(u a v)=w(a)$ for all $a \in A$ and $u, v \in \mathcal{U}$.

Lemma 2.4. Let $\alpha, \beta \in \mathcal{R}$ and $w \in \mathcal{A}$ all be bi-invariant. Then $\alpha * \beta$, $\widehat{\alpha}$, and $w \circledast \alpha$ are bi-invariant.

Proof. We will verify the claim for $w \circledast \alpha$; the others are similar.

$$
\begin{aligned}
(w \circledast \alpha)(u a v) & =\sum_{r \in R} w(\text { ruav }) \alpha(r)=\sum_{r \in R} w(\text { rua }) \alpha(r) \\
& =\sum_{s \in R} w(s a) \alpha\left(s u^{-1}\right)=\sum_{s \in R} w(s a) \alpha(s)=(w \circledast \alpha)(a),
\end{aligned}
$$

where we made use of the change of variables $s=r u, u \in \mathcal{U}$.

\section{Some Computations}

Suppose $w \in \mathcal{A}_{0}$ is bi-invariant. By making the change of variable $a \leftrightarrow-a$ and using bi-invariance, the inverse Fourier transform of $w$ is

$$
\widetilde{w}(r)=\frac{1}{|A|} \sum_{a \in A} w(a) \chi(r a) .
$$

Lemma 3.1. Suppose $w \in \mathcal{A}_{0}$ is bi-invariant. Then $\widetilde{w} \in \mathcal{R}_{0}$ is bi-invariant and $\chi \circledast \widetilde{w}=w$.

Proof. Let us begin by verifying that $\widetilde{w} \in \mathcal{R}_{0}$ :

$$
\begin{aligned}
|A| \sum_{r \in R} \widetilde{w}(r) & =\sum_{r \in R} \sum_{a \in A} w(a) \chi(r a) \\
& =\sum_{a \in A} w(a) \sum_{r \in R} \chi(r a)=|R| w(0)=0,
\end{aligned}
$$

where we simplified the summation using Lemma 2.1 for $S=R a$ :

$$
\sum_{r \in R} \chi(r a)= \begin{cases}|R|, & a=0, \\ 0, & a \neq 0 .\end{cases}
$$

By the change of variable $b=v a, v \in \mathcal{U}$, one sees that $\widetilde{w}(r v)=\widetilde{w}(r)$. For $u \in \mathcal{U},|A| \widetilde{w}(u r)=\sum_{a \in A} w(a) \chi^{u}(r a)$. Because $u$ is a unit, $\chi^{u}$ is another generating character for $A$. As such, it must equal ${ }^{u^{\prime}} \chi$ for some unit $u^{\prime} \in \mathcal{U}$. Then $|A| \widetilde{w}(u r)=$ $\sum_{a \in A} w(a) \chi\left(r a u^{\prime}\right)$. Using the change of variable $b=a u^{\prime}$, we conclude that $\widetilde{w}(u r)=$ $\widetilde{w}(r)$.

Finally, using bi-invariance of $w$ so that $w(-b)=w(b)$,

$$
\begin{aligned}
|A|(\chi \circledast \widetilde{w})(a) & =\sum_{r \in R} \chi(r a) \sum_{b \in A} w(b) \chi(-r b) \\
& =\sum_{b \in A} w(b) \sum_{r \in R} \chi(r(a-b))=|A| w(a),
\end{aligned}
$$

where we used $|A|=|R|$ and Lemma 2.1 with $S=R(a-b)$. 
A particular example of a bi-invariant weight on a Frobenius bimodule is the homogeneous weight $w_{\text {Hom }}$. The existence of the homogeneous weight on any Frobenius bimodule was proved in [6] and [9]. We will need the features of the homogeneous weight listed in the next theorem.

THEOREM $3.2(\mathbf{8})$. Suppose $w_{\mathrm{Hom}}$ is the homogeneous weight on a Frobenius bimodule A. Then

(1) $w_{\text {Hom }}$ is expressible in terms of the generating character $\chi$ of $A$ :

$$
w_{\text {Hom }}(a)=1-\frac{1}{|\mathcal{U}|} \sum_{u \in \mathcal{U}} \chi(u a)
$$

(2) $w_{\text {Hom }}$ is bi-invariant.

Define $\varepsilon \in \mathcal{R}_{0}$ by

$$
\varepsilon(r)= \begin{cases}-1 /|\mathcal{U}|, & r \in \mathcal{U}, \\ 1, & r=0, \\ 0, & \text { otherwise. }\end{cases}
$$

Lemma 3.3. The Fourier transform of $\varepsilon$ is $w_{\mathrm{Hom}}$, the homogeneous weight of $A$. That is, $\chi \circledast \varepsilon=w_{\mathrm{Hom}}$.

Proof. Calculate and make use of Theorem 3.2

$$
\begin{aligned}
(\chi \circledast \varepsilon)(a) & =\sum_{r \in R} \chi(r a) \varepsilon(r) \\
& =1-(1 /|\mathcal{U}|) \sum_{u \in \mathcal{U}} \chi(u a)=w_{\text {Hom }}(a) .
\end{aligned}
$$

We quote the following lemma:

Lemma 3.4 (13. Prop. 5.1]). For cyclic right submodules of $A, a R=b R$ if and only if $a \mathcal{U}=b \mathcal{U}$.

The set $P=\{a R: a \in A\}$ of all cyclic right submodules of $A$ is a partially ordered set (poset) under inclusion $\subseteq$. Denote the Möbius function of $P$ by $\mu$. Then $\mu$ is characterized by the following properties:

- $\mu(a R, c R)=0$ when $a R \nsubseteq c R$;

- $\mu(a R, a R)=1$;

- $\sum_{a R \subseteq b R \subseteq c R} \mu(a R, b R)=0$, where $b R$ varies for fixed $a R \subsetneq c R$.

For further details about posets and their Möbius functions, see $\mathbf{1 2}$.

The next result appears in $[\mathbf{8}$.

Lemma 3.5. The Möbius function of $P$ has $\mu(0, a R)=\sum_{b \in a \mathcal{U}} \chi(b)$.

Proof. Define $g: P \rightarrow \mathbb{C}$ by $g(a R)=\sum_{b \in a \mathcal{U}} \chi(b)$. We verify that $g$ satisfies the properties of $\mu(0, \cdot)$.

Certainly $g(0)=\chi(0)=1$. For fixed $c R \neq 0$, use Lemma 2.1

$$
\sum_{b R \subseteq c R} g(b R)=\sum_{b \mathcal{U} \subseteq c R} \sum_{a \in b \mathcal{U}} \chi(a)=\sum_{a \in c R} \chi(a)=0 .
$$


Suppose $w \in \mathcal{A}_{0}$ is bi-invariant. Consider $w$ that satisfy:

$$
\sum_{a R \subseteq S} w(a) \mu(0, a R) \neq 0,
$$

for all nonzero right submodules $S \subseteq A$.

Lemma 3.6. Let $w \in \mathcal{A}_{0}$ be bi-invariant. Then (3.1) is equivalent to

$$
\sum_{a \in S} w(a) \chi(a) \neq 0, \quad \text { for all nonzero right submodules } S \subseteq A .
$$

Proof. The group of units $\mathcal{U}$ acts on $A$ on the right by scalar multiplication. Any right submodule $S$ of $A$ is invariant under this action. Thus $S$ is the disjoint union of right $\mathcal{U}$-orbits. Break up the sum over $S$ into the sum over these right $\mathcal{U}$-orbits. Then, by Lemma 3.5 .

$$
\begin{aligned}
\sum_{a \in S} w(a) \chi(a) & =\sum_{a \mathcal{U} \subseteq S} \sum_{b \in a \mathcal{U}} w(b) \chi(b)=\sum_{a \mathcal{U} \subseteq S} w(a) \sum_{b \in a \mathcal{U}} \chi(b) \\
& =\sum_{a R \subseteq S} w(a) \mu(0, a R) .
\end{aligned}
$$

For later use in the proof of Lemma 3.8, we examine factorizations $s t=r$ with $R r=R t$. Fix $r \in R$. Suppose $s t=r$ and $R r=R t$. Lemma 3.4 implies that $t=u r$ for some $u \in \mathcal{U}$. Then $r=s t=s u r$, so that $(s u-1) r=0$. That is, $s u-1 \in \operatorname{ann}_{\mathrm{lt}}(r)$, where $\operatorname{ann}_{\mathrm{lt}}(r)=\{q \in R: q r=0\}$ is the left annihilator of $r$ in $R$; $\operatorname{ann}_{1 \mathrm{t}}(r)$ is a left ideal in $R$. In summary, if $s t=r$ and $R r=R t$, then there exist $u \in \mathcal{U}$ and $q \in \operatorname{ann}_{\mathrm{lt}}(r)$ with $s=(1+q) u^{-1}$ and $t=u r$. Conversely, if $u \in \mathcal{U}$ and $q \in \operatorname{ann}_{\mathrm{lt}}(r)$, then setting $s=(1+q) u^{-1}$ and $t=u r$ yields a factorization $s t=r$ with $R r=R t$.

It is possible that different choices of $u$ and $q$ may yield the same factors $s$ and $t$. The next lemma gives a precise description of the possibilities. Fix $r \in R$. Define $\mathcal{F}(r)=\{(s, t) \in R \times R: s t=r$ and $R r=R t\}$, the set of all factorizations of $r$ with $R r=R t$. Define $\operatorname{Stab}(r)=\{u \in \mathcal{U}: u r=r\}$, the stabilizer subgroup of $r$ under the left action of $\mathcal{U}$ on $R$. Define

$$
F: \operatorname{ann}_{\mathrm{lt}}(r) \times \mathcal{U} \rightarrow \mathcal{F}(r), \quad F(q, u)=\left((1+q) u^{-1}, u r\right) .
$$

LEMma 3.7. The function $F: \operatorname{ann}_{1 \mathrm{t}}(r) \times \mathcal{U} \rightarrow \mathcal{F}(r)$ is onto and, for every $(s, t) \in \mathcal{F}(r),\left|F^{-1}(s, t)\right|=|\operatorname{Stab}(r)|$.

Proof. The discussion in the preceding paragraphs shows that $F$ is onto. For any $(s, t) \in \mathcal{F}(r)$, pick one point $\left(q_{0}, u_{0}\right) \in F^{-1}(s, t)$. If $(q, u)$ is any other point in $F^{-1}(s, t)$, then $s=\left(1+q_{0}\right) u_{0}^{-1}=(1+q) u^{-1}$ and $t=u_{0} r=u r$. From the latter equation, we see that $u_{0}^{-1} u \in \operatorname{Stab}(r)$.

Define $\phi: F^{-1}(s, t) \rightarrow \operatorname{Stab}(r)$ by $\phi(q, u)=u_{0}^{-1} u$, and define $\psi: \operatorname{Stab}(r) \rightarrow$ $F^{-1}(s, t)$ by $\psi(z)=\left(\left(1+q_{0}\right) z-1, u_{0} z\right)$. One readily verifies that $\phi$ and $\psi$ are inverse functions.

The next computation is the key technical result in the paper.

Lemma 3.8. Suppose $w \in \mathcal{A}_{0}$ is bi-invariant and satisfies (3.1) for all nonzero right submodules $S \subseteq A$. Then there exists $\gamma \in \mathcal{R}_{0}$ that is bi-invariant and satisfies $\widetilde{w} * \gamma=\varepsilon$. 
Proof. We define $\gamma$ in stages, starting on the group of units $\mathcal{U}$ in $R$, by solving the equation

$$
\varepsilon(r)=\sum_{s t=r} \widetilde{w}(s) \gamma(t)
$$

If $r=u \in \mathcal{U}$, then $s t=u$ implies that $s$ and $t$ are in $\mathcal{U}$. Equation (3.2) then becomes $-1 /|\mathcal{U}|=\sum_{t \in \mathcal{U}} \widetilde{w}\left(r t^{-1}\right) \gamma(t)=|\mathcal{U}| \widetilde{w}(1) \gamma(1)$. We have used the biinvariance of $\widetilde{w}$ and the desired bi-invariance of $\gamma$. Note that $\widetilde{w}(1) \neq 0$, this being the $S=A$ case of (3.1). Thus, by defining $\gamma(t)=\gamma(1)=-1 /\left(|\mathcal{U}|^{2} \widetilde{w}(1)\right)$, we solve (3.2) when $r=u$, and that portion of $\gamma$ that has been defined is bi-invariant.

As a recursive step, suppose a solution $\gamma$ has been defined for various $r \in R$ such that $\gamma$ is bi-invariant; i.e., if $\gamma(r)$ is defined, then $\gamma(u r)$ and $\gamma(r u)$ are also defined for all $u \in \mathcal{U}$, and $\gamma(u r)=\gamma(r u)=\gamma(r)$ for all $u \in \mathcal{U}$. Now let $r \in R$ be any element, neither a unit nor 0 , such that $R r$ is maximal among all principal left ideals of $R$ where $\gamma$ is not defined on $\mathcal{U} r$. Consider (3.2) for this choice of $r$.

Any solution of the equation $s t=r$ has the property that $R r \subseteq R t$. If $R r \subsetneq R t$, then the maximality property of $R r$ implies that $\gamma(t)$ is already defined. Then (3.2) becomes

$$
0=\sum_{\substack{s t=r \\
R r \subsetneq R t}} \widetilde{w}(s) \gamma(t)+\sum_{\begin{array}{c}
s t=r \\
R r=R t
\end{array}} \widetilde{w}(s) \gamma(t) .
$$

We analyze the sum where $R r=R t$. Using Lemma 3.7 and the bi-invariance of $\widetilde{w}$ and $\gamma$, we see that

$$
\begin{aligned}
|\operatorname{Stab}(r)| \sum_{\substack{s t=r \\
R r=R t}} \widetilde{w}(s) \gamma(t) & =\sum_{q \in \operatorname{ann}_{\mathrm{lt}}(r), u \in \mathcal{U}} \widetilde{w}\left((1+q) u^{-1}\right) \gamma(u r) \\
& =|\mathcal{U}| \gamma(r) \sum_{q \in \operatorname{ann}_{\mathrm{lt}}(r)} \widetilde{w}(1+q) .
\end{aligned}
$$

The last summation simplifies in the following way:

$$
\begin{aligned}
|A| \sum_{q \in \mathrm{ann}_{\mathrm{lt}}(r)} \widetilde{w}(1+q) & =\sum_{q \in \mathrm{ann}_{\mathrm{lt}}(r)} \sum_{a \in A} w(a) \chi((1+q) a) \\
& =\sum_{a \in A} w(a) \chi(a) \sum_{q \in \operatorname{ann}_{\mathrm{lt}}(r)} \chi(q a) .
\end{aligned}
$$

The summation $\sum_{q \in \operatorname{ann}_{1 \mathrm{t}}(r)} \chi(q a)$ is a sum over the left submodule $\operatorname{ann}_{1 \mathrm{t}}(r) a \subseteq A$. Because $\chi$ is a generating character for $A$, this sum vanishes (Lemma 2.1) unless the submodule $\operatorname{ann}_{\mathrm{lt}}(r) a=0$, in which case the sum equals $\left|\operatorname{ann}_{\mathrm{lt}}(r)\right|$.

For $r \in R$, define $S_{r}=\left\{a \in A: \operatorname{ann}_{\mathrm{lt}}(r) a=0\right\} ; S_{r}$ is a right submodule of $A$. Continuing the simplification from above, we see that

$$
|A| \sum_{q \in \operatorname{ann}_{\mathrm{lt}}(r)} \widetilde{w}(1+q)=\left|\operatorname{ann}_{\mathrm{lt}}(r)\right| \sum_{a \in S_{r}} w(a) \chi(a) .
$$

By the $S=S_{r}$ case of (3.1) and Lemma 3.6. we see that $\sum_{a \in S_{r}} w(a) \chi(a) \neq 0$ and hence $\sum_{q \in \operatorname{ann}_{\text {lt }}(r)} \widetilde{w}(1+q) \neq 0$. This allows us to solve for $\gamma(r)$ in terms of previously defined values of $\gamma$ :

$$
\gamma(r)=-\left(|\operatorname{Stab}(r)| \sum_{\substack{s t=r \\ R r \subsetneq R t}} \widetilde{w}(s) \gamma(t)\right) /\left(|\mathcal{U}| \sum_{q \in \operatorname{ann}_{1 \mathrm{t}}(r)} \widetilde{w}(1+q)\right) .
$$


This formula gives the same value for $\gamma(r u), u \in \mathcal{U}$, and the entire derivation is the same if $r$ is replaced by $u r$. Thus $\gamma$ is bi-invariant.

Proceeding recursively, we eventually arrive at the case $r=0$. Then (3.2) becomes

$$
1=\sum_{\substack{s t=0 \\ t \neq 0}} \widetilde{w}(s) \gamma(t)+\sum_{s \in R} \widetilde{w}(s) \gamma(0) .
$$

Because $\widetilde{w} \in \mathcal{R}_{0}$, the coefficient $\sum_{s \in R} \widetilde{w}(s)$ of $\gamma(0)$ vanishes. Thus, the equation puts no restriction on $\gamma(0)$. By setting $\gamma(0)=-\sum_{r \neq 0} \gamma(r)$, we have $\gamma \in \mathcal{R}_{0}$.

Why does $(\widetilde{w} * \gamma)(0)=1$ ? We know $\widetilde{w} \in \mathcal{R}_{0}$, and we have already shown that $\gamma \in \mathcal{R}_{0}$ and $(\widetilde{w} * \gamma)(r)=\varepsilon(r)$ for all $r \neq 0$. Since $\widetilde{w} * \gamma$ and $\varepsilon$ belong to $\mathcal{R}_{0}$, we conclude that their values at $r=0$ also agree.

Corollary 3.9. Suppose $w \in \mathcal{A}_{0}$ is bi-invariant and satisfies (3.1) for all nonzero right submodules $S \subseteq A$. Then $w \circledast \gamma=w_{\text {Hom }}$.

Proof. Use Lemmas 2.2, 3.1, 3.3, and 3.8, Then, $w \circledast \gamma=(\chi \circledast \widetilde{w}) \circledast \gamma=$ $\chi \circledast(\widetilde{w} * \gamma)=\chi \circledast \varepsilon=w_{\text {Hom }}$.

\section{The Main Theorem}

As in earlier sections, $R$ is a finite ring with 1 and $A$ is a Frobenius bimodule over $R$. An $R$-linear code over $A$ of length $n$ is a left $R$-submodule $C \subseteq A^{n}$. A function $w \in \mathcal{A}_{0}$ defines a weight on $A^{n}$ (and, by restriction, on $C$ ) by $w(x)=\sum_{i=1}^{n} w\left(x_{i}\right)$, where $x=\left(x_{1}, x_{2}, \ldots, x_{n}\right) \in A^{n}$. An injective $R$-linear homomorphism $f: C \rightarrow A^{n}$ is a $w$-isometry if $w(f(x))=w(x)$ for all $x \in C$.

Proposition 4.1. Suppose $f: C \rightarrow A^{n}$ is a $w$-isometry. Then $f$ is a $w \circledast \alpha$ isometry for any $\alpha \in \mathcal{R}$.

Proof. For $x \in C$,

$$
\begin{aligned}
(w \circledast \alpha)(f(x)) & =\sum_{r \in R} w(r f(x)) \alpha(r)=\sum_{r \in R} w(f(r x)) \alpha(r) \\
& =\sum_{r \in R} w(r x) \alpha(r)=(w \circledast \alpha)(x) .
\end{aligned}
$$

For units $u_{1}, u_{2}, \ldots, u_{n} \in \mathcal{U}$ and a permutation $\sigma$ of $\{1,2, \ldots, n\}$, the left $R$ module homomorphism $T: A^{n} \rightarrow A^{n}$ defined by

$$
T\left(x_{1}, x_{2}, \ldots, x_{n}\right)=\left(x_{\sigma(1)} u_{1}, x_{\sigma(2)} u_{2}, \ldots, x_{\sigma(n)} u_{n}\right),
$$

$\left(x_{1}, x_{2}, \ldots, x_{n}\right) \in A^{n}$, is called a monomial transformation of $A^{n}$. If $w$ is biinvariant, then any monomial transformation is a $w$-isometry.

Definition 4.2. A bi-invariant weight $w$ on a Frobenius bimodule $A$ has the extension property if the following holds: for any linear code $C \subseteq A^{n}$ and any $w$-isometry $f: C \rightarrow A^{n}$, the isometry $f$ extends to a monomial transformation of $A^{n}$.

An example of a bi-invariant weight that has the extension property is the homogeneous weight $w_{\text {Hom }}$ :

Theorem 4.3 ([6. Theorem 4.10]). Any Frobenius bimodule A has the extension property with respect to $w_{\mathrm{Hom}}$. 
ThEOREM 4.4. Suppose $A$ is a Frobenius bimodule over $R$ and $w$ is a biinvariant weight on $A$. If $w$ satisfies (3.1) for all nonzero right submodules $S \subseteq A$, then $w$ has the extension property.

Proof. Suppose $C \subseteq A^{n}$ is a linear code and $f: C \rightarrow A^{n}$ is a $w$-isometry. Then $f$ is an isometry with respect to $w \circledast \gamma=w_{\text {Hom }}$, Corollary 3.9 and Proposition 4.1. By Theorem 4.3, $w_{\text {Hom }}$ has the extension property, so $f$ extends to a monomial transformation, as desired.

COROllary 4.5. Suppose $R$ is a finite Frobenius ring and $w$ is a bi-invariant weight on $R$. If $w$ satisfies (3.1) for all nonzero right ideals $S \subseteq R$, then $w$ has the extension property.

Remark 4.6. Corollary 4.5 generalizes to all finite Frobenius rings the condition proved for finite principal ideal rings in 4, Theorem 4.4].

\section{Example}

We illustrate Theorem 4.4 with an example.

EXAmPLE 5.1. Let $R=U_{2}\left(\mathbb{F}_{2}\right)$ be the ring of $2 \times 2$ upper-triangular matrices over $\mathbb{F}_{2}$. The ring $R$ is an algebra over $\mathbb{F}_{2}$ of dimension 3 , so that $|R|=8$. When $r \in R$ is the matrix

$$
r=\left[\begin{array}{ll}
a & b \\
0 & c
\end{array}\right]
$$

we will denote the element $r$ with the notation $r=[a, b, c]$. It is known that the ring $R$ is not Frobenius.

The Frobenius bimodule $A=\widehat{R}$ is a vector space over $\mathbb{F}_{2}$ of dimension 3 . For $\pi \in A$, we will use the notation $\pi=(x, y, z)$, where the evaluation $\pi(r)$, $r=[a, b, c] \in R$, is

$$
\pi(r)=(-1)^{a x+b y+c z} .
$$

The following formulas describe the scalar multiplications on the bimodule $A$ over $R$. As above, $r=[a, b, c]$ and $\pi=(x, y, z)$ :

$$
\begin{aligned}
{ }^{r} \pi & =(a x+b y, c y, c z), \\
\pi^{r} & =(a x, a y, b y+c z) .
\end{aligned}
$$

In order to understand (3.1), we need to know the right submodules of $A$. For $\pi=(x, y, z) \in A$, we will denote the right submodule generated by $\pi$ as $(x, y, z) R$. By straight-forward computations using the formulas above, we have the following list of right submodules of $A$. The module $A$ is not a cyclic module, while all its proper submodules are cyclic.

$$
\begin{aligned}
& \operatorname{dim} 0: 0=(0,0,0) R \\
& \operatorname{dim} 1: \\
& \operatorname{dim} 2:(0,0,1) R,(1,0,0) R ; \\
& \operatorname{dim} 3: \quad A \text { itself. }
\end{aligned}
$$

Also, $(1,0,0) R \subset(1,0,1) R$, and $(0,0,1) R$ is contained in every submodule of dimension 2. See Figure 5.1, where some containments are dotted because $A$ is not itself a cyclic right module. 
FiguRE 5.1. The cyclic right submodules of $A$.

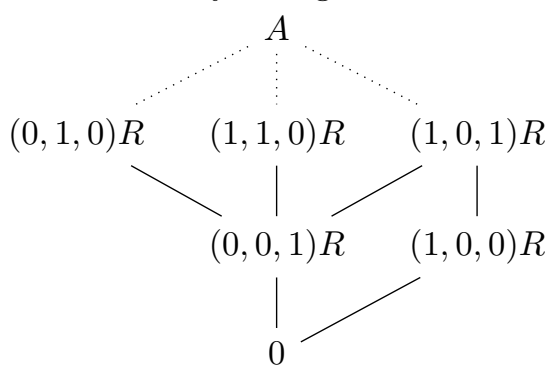

By using the containments just described, we calculate that the values of the Möbius function $\mu(0, a R)$ are as follows:

\begin{tabular}{c|cccccc}
$a$ & 0 & $(0,0,1)$ & $(1,0,0)$ & $(0,1,0)$ & $(1,0,1)$ & $(1,1,0)$ \\
\hline$\mu(0, a R)$ & 1 & -1 & -1 & 0 & 1 & 0
\end{tabular}.

Theorem 4.4 applies to bi-invariant weights. The description above of the right cyclic submodules implies that $(0,1,0) \mathcal{U}=(0,1,1) \mathcal{U}$ and $(1,1,0) \mathcal{U}=(1,1,1) \mathcal{U}$, with all other right $\mathcal{U}$-orbits being distinct. Similar computations of left $\mathcal{U}$-orbits show that all the left $\mathcal{U}$-orbits are distinct except for $\mathcal{U}(0,1,0)=\mathcal{U}(1,1,0)$ and $\mathcal{U}(0,1,1)=\mathcal{U}(1,1,1)$. Thus, a weight $w$ on $A$ is bi-invariant if and only if

$$
w(0,1,0)=w(0,1,1)=w(1,1,0)=w(1,1,1) .
$$

Theorem 4.4 says that a bi-invariant weight $w$ has the extension property when the following expressions are nonzero:

\begin{tabular}{l|l} 
right module $S$ & expression in (3.1) \\
\hline$(0,0,1) R$ & $-w(0,0,1)$ \\
$(1,0,0) R$ & $-w(1,0,0)$ \\
$(0,1,0) R$ & $-w(0,0,1)$ \\
$(1,0,1) R$ & $-w(0,0,1)-w(1,0,0)+w(1,0,1)$ \\
$(1,1,0) R$ & $-w(0,0,1)$ \\
$A$ & $-w(0,0,1)-w(1,0,0)+w(1,0,1)$
\end{tabular}

In particular, the value of $w(0,1,0)$ is irrelevant.

\section{Other Alphabets}

In this section we consider the extension problem for certain other alphabets.

Suppose $R$ is a finite ring with 1 and $A$ is a finite unital left $R$-module. Let $w$ be a weight on $A$, i.e., a function $w: A \rightarrow \mathbb{C}$ with $w(0)=0$. Then $w$ extends to a weight on $A^{n}$ by $w(x)=\sum_{i=1}^{n} w\left(x_{i}\right)$, where $x=\left(x_{1}, x_{2}, \ldots, x_{n}\right) \in A^{n}$. The group of all invertible homomorphisms $\phi: A \rightarrow A$ is denoted $\mathrm{GL}_{R}(A)$, and $\phi$ will be written with inputs on the left, so that $(r a) \phi=r(a \phi)$ for all $r \in R$ and $a \in A$. The right symmetry group of $w$ on $A$ is

$$
G_{\mathrm{rt}}=\left\{\phi \in \mathrm{GL}_{R}(A): w(a \phi)=w(a), a \in A\right\} .
$$

A $G_{\mathrm{rt}}$-monomial transformation of $A^{n}$ is any invertible homomorphism $T: A^{n} \rightarrow$ $A^{n}$ of the form

$$
\left(x_{1}, x_{2}, \ldots, x_{n}\right) T=\left(x_{\sigma(1)} \phi_{1}, x_{\sigma(2)} \phi_{2}, \ldots, x_{\sigma(n)} \phi_{n}\right),
$$


for $\left(x_{1}, x_{2}, \ldots, x_{n}\right) \in A^{n}$, where $\sigma$ is a permutation of $\{1,2, \ldots, n\}$ and $\phi_{i} \in G_{\mathrm{rt}}$. Observe that a $G_{\mathrm{rt}}$-monomial transformation preserves $w: w(x T)=w(x)$ for all $x \in A^{n}$.

A weight $w$ on $A$ has the extension property if, for any left $R$-linear code (submodule) $C \subseteq A^{n}$ and any injective homomorphism $f: C \rightarrow A^{n}$ of left $R$ modules that preserves $w, w(c)=w(f(c)), c \in C$, it follows that $f$ extends to a $G_{\mathrm{rt}}$-monomial transformation of $A^{n}$.

We will prove that the extension property holds for certain alphabets. Let $A$ be a finite unital left $R$-module that is pseudo-injective and such that its $\operatorname{socle} \operatorname{soc}(A)$ is a cyclic left $R$-module. Recall that a left $R$-module is pseudo-injective if for any left $R$-submodule $B \subseteq A$ and any injective homomorphism $\phi: B \rightarrow A$ of left $R$-modules, $\phi$ extends to a homomorphism $\tilde{\phi}: A \rightarrow A$ of left $R$-modules. It follows from [1, Proposition 3.2] that there exists an extension $\tilde{\phi}$ that is injective, hence invertible. The socle $\operatorname{soc}(A)$ of $A$ is the left submodule generated by all simple left $R$-submodules of $A$. The hypothesis that $\operatorname{soc}(A)$ is cyclic implies that $A$ injects into $\widehat{R}$ as a left $R$-module [15, Proposition 5.3].

Theorem 6.1. Suppose $R$ is a finite ring with 1 and $A$ is a finite unital left $R$-module that is pseudo-injective and has $\operatorname{soc}(A)$ cyclic. Fix an injection of $A$ into $\widehat{R}$, and view $A$ as a left submodule of $\widehat{R}$. Let $w$ be a weight on $A$, and assume that $w$ is the restriction to $A$ of a bi-invariant weight on $\widehat{R}$ that satisfies (3.1). Then the weight $w$ on the alphabet $A$ satisfies the extension property.

Before proving Theorem 6.1 we state the following lemma.

Lemma 6.2. Suppose $R$ is a finite ring with $1, I$ is a finite injective left module over $R$, and $A \subseteq I$ is a left $R$-submodule. Then every element of $\mathrm{GL}_{R}(A)$ extends to an element of $\mathrm{GL}_{A}(I)$.

Proof. This is essentially [1, Proposition 3.2], discussed above. Any injective module is, a fortiori, pseudo-injective. Injections $A \rightarrow A \subseteq I$ then extend to injections $I \rightarrow I$.

Proof of Theorem 6.1. Suppose $C \subseteq A^{n}$ is a left $R$-linear code and $f: C \rightarrow$ $A^{n}$ is an injective homomorphism that preserves $w$. Because $A \subseteq \widehat{R}$, we can view $C \subseteq \widehat{R}^{n}$ as an $R$-linear code over the alphabet $\widehat{R}$ and $f: C \rightarrow \widehat{R}^{n} ; f$ still preserves $w$, now viewed as a bi-invariant weight on $\widehat{R}$ that satisfies (3.1). By Theorem 4.4. $f$ extends to a monomial transformation $T: \widehat{R}^{n} \rightarrow \widehat{R}^{n}$.

Write the components of $f: C \rightarrow A^{n}$ as $f=\left(f_{1}, f_{2} \ldots, f_{n}\right), f_{i}: C \rightarrow A$. The fact that $f$ extends to the monomial transformation $T$ means there exist a permutation $\sigma$ of $\{1,2, \ldots, n\}$ and units $u_{i} \in \mathcal{U}(R)$ such that $f_{i}(x)=x_{\sigma(i)} u_{i}$, for $x=\left(x_{1}, x_{2}, \ldots, x_{n}\right) \in C$. Because $f: C \rightarrow A^{n}$, this means that $x_{\sigma(i)} u_{i} \in A$ for $x \in C$.

Let $C_{i}=\left\{x_{i}: x \in C\right\}$ be the projection of $C \subseteq A^{n}$ on the $i$ th coordinate; $C_{i} \subseteq A$ is an $R$-submodule. The extendability of $f$ implies that right multiplication by $u_{i}$ injects $C_{\sigma(i)} \rightarrow A$. Because $A$ is pseudo-injective, this injection extends to an injection $A \rightarrow A$, i.e., to an element $\phi_{i} \in \mathrm{GL}_{R}(A)$. Thus, $f_{i}(x)=x_{\sigma(i)} \phi_{i}, x \in C$.

The last thing we need to prove is that $\phi_{i} \in G_{\mathrm{rt}}$, i.e., that $\phi_{i}$ preserves $w$ on $A$. This follows from Lemma 6.2. Indeed, $\widehat{R}$ is always an injective module, so $\phi_{i} \in \mathrm{GL}_{R}(A)$ extends to an element of $\mathrm{GL}_{R}(\widehat{R})$. But $\mathrm{GL}_{R}(\widehat{R})=\mathcal{U}(R)$, and 
$w$ is bi-invariant as a weight on $\widehat{R}$, so every element of $\mathrm{GL}_{R}(\widehat{R})$ preserves $w$. By restriction, every element of $\mathrm{GL}_{R}(A)$ preserves $w$ on $A$.

\section{References}

[1] H. Q. Dinh and S. R. López-Permouth, On the equivalence of codes over finite rings, Appl. Algebra Engrg. Comm. Comput. 15 (2004), no. 1, 37-50, DOI 10.1007/s00200-004-0149-5. MR2142429

[2] M. Greferath and T. Honold, On weights allowing for MacWilliams equivalence theorem, Proceedings of the Fourth International Workshop in Optimal Codes and Related Topics (Pamporovo, Bulgaria), 2005, pp. 182-192.

[3] M. Greferath and T. Honold, Monomial extensions of isometries of linear codes II: Invariant weight functions on $Z_{m}$, Proceedings of the Tenth International Workshop in Algebraic and Combinatorial Coding Theory (ACCT-10) (Zvenigorod, Russia), 2006, pp. 106-111.

[4] M. Greferath, T. Honold, C. Mc Fadden, J. A. Wood, and J. Zumbrägel, MacWilliams' extension theorem for bi-invariant weights over finite principal ideal rings, J. Combin. Theory Ser. A 125 (2014), 177-193, DOI 10.1016/j.jcta.2014.03.005. MR3207471

[5] M. Greferath, C. Mc Fadden, and J. Zumbrägel, Characteristics of invariant weights related to code equivalence over rings, Des. Codes Cryptogr. 66 (2013), no. 1-3, 145-156, DOI 10.1007/s10623-012-9671-9. MR3016561

[6] M. Greferath, A. Nechaev, and R. Wisbauer, Finite quasi-Frobenius modules and linear codes, J. Algebra Appl. 3 (2004), no. 3, 247-272, DOI 10.1142/S0219498804000873. MR2096449

[7] M. Greferath and S. E. Schmidt, Finite-ring combinatorics and MacWilliams' equivalence theorem, J. Combin. Theory Ser. A 92 (2000), no. 1, 17-28, DOI 10.1006/jcta.1999.3033. MR 1783936

[8] T. Honold, Characterization of finite Frobenius rings, Arch. Math. (Basel) 76 (2001), no. 6, 406-415, DOI 10.1007/PL00000451. MR1831096

[9] A. A. Nechaev and T. Khonol'd, Fully weighted modules and representations of codes (Russian, with Russian summary), Problemy Peredachi Informatsii 35 (1999), no. 3, 18-39; English transl., Problems Inform. Transmission 35 (1999), no. 3, 205-223. MR1730800

[10] J. MacWilliams, Error-correcting codes for multiple-level transmission, Bell System Tech. J. 40 (1961), 281-308, DOI 10.1002/j.1538-7305.1961.tb03986.x. MR0141541

[11] F. J. MacWilliams, COMBINATORIAL PROBLEMS OF ELEMENTARY ABELIAN GROUPS, ProQuest LLC, Ann Arbor, MI, 1962. Thesis (Ph.D.)-Radcliffe College. MR2939359

[12] R. P. Stanley, Enumerative combinatorics. Vol. I, The Wadsworth \& Brooks/Cole Mathematics Series, Wadsworth \& Brooks/Cole Advanced Books \& Software, Monterey, CA, 1986. With a foreword by Gian-Carlo Rota. MR847717

[13] J. A. Wood, Duality for modules over finite rings and applications to coding theory, Amer. J. Math. 121 (1999), no. 3, 555-575. MR1738408

[14] J. A. Wood, Weight functions and the extension theorem for linear codes over finite rings, Finite fields: theory, applications, and algorithms (Waterloo, ON, 1997), Contemp. Math., vol. 225, Amer. Math. Soc., Providence, RI, 1999, pp. 231-243, DOI 10.1090/conm/225/03225. MR 1650644

[15] J. A. Wood, Foundations of linear codes defined over finite modules: the extension theorem and the MacWilliams identities, Codes over rings, Ser. Coding Theory Cryptol., vol. 6, World Sci. Publ., Hackensack, NJ, 2009, pp. 124-190, DOI 10.1142/9789812837691_0004. MR2850303 
Department of Mathematics and Systems Analysis, Aalto University, P.O. Box 11100, FI-00076 AALTO, Finland

Email address: oliver.gnilke@aalto.fi

Department of Mathematics and Systems Analysis, Aalto University, P.O. Box 11100, FI-00076 Aalto, Finland

Email address: marcus.greferath@aalto.fi

ZJU-UiUC Institute, Zhejiang University, 718 East Haizhou Road, Haining, 314400 , CHINA

Email address: honold@zju.edu.cn

Department of Mathematics, Western Michigan University, 1903 W. Michigan AvEnue, Kalamazoo, Michigan 49008-5248

Email address: jay.wood@wmich.edu

Faculty of Computer Science and Mathematics, University of Passau, Innstrasse 33, 94032 Passau, Germany

Email address: jens.zumbraegel@uni-passau.de 